\title{
In vitro antiplasmodial activity of cepharanthine
}

\author{
Camille Desgrouas ${ }^{1}$, Charles Chapus ${ }^{2}$, Jérôme Desplans ${ }^{1}$, Christelle Travaille ${ }^{3}$, Aurélie Pascual ${ }^{4}$, Béatrice Baghdikian ${ }^{5}$, \\ Evelyne Ollivier ${ }^{5}$, Daniel Parzy ${ }^{1}$ and Nicolas Taudon ${ }^{2 *}$
}

\begin{abstract}
Background: New classes of anti-malarial drugs are needed to control the alarming Plasmodium falciparum resistance toward current anti-malarial therapy. The ethnopharmacological approach allows the discovery of original chemical structures from the vegetable biodiversity. Previous studies led to the selection of a bisbenzylisoquinoline, called cepharanthine and isolated from a Cambodian plant: Stephania rotunda. Cepharanthine could exert a mechanism of action different from commonly used drugs. Potential plasmodial targets are reported here.
\end{abstract}

Methods: To study the mechanism of action of cepharanthine, a combined approach using phenotypic and transcriptomic techniques was undertaken.

Results: Cepharanthine blocked P. falciparum development in ring stage. On a culture of synchronized ring stage, the comparisons of expression profiles showed that the samples treated with $5 \mu \mathrm{M}$ of cepharanthine $\left(\mathrm{IC}_{90}\right)$ were significantly closer to the initial controls than to the final ones. After a two-way ANOVA ( $p$-value $<0.05$ ) on the microarray results, 1,141 probes among 9,722 presented a significant differential expression.

A gene ontology analysis showed that the Maurer's clefts seem particularly down-regulated by cepharanthine. The analysis of metabolic pathways showed an impact on cell-cell interactions (cytoadherence and rosetting), glycolysis and isoprenoid pathways. Organellar functions, more particularly constituted by apicoplast and mitochondrion, are targeted too.

Conclusion: The blockage at the ring stage by cepharanthine is described for the first time. Transcriptomic approach confirmed that cepharanthine might have a potential innovative antiplasmodial mechanism of action. Thus, cepharanthine might play an ongoing role in the progress on anti-malarial drug discovery efforts.

Keywords: Stephania rotunda, Cepharanthine, Plasmodium falciparum, Antiplasmodial activity, Transcriptomic analysis

\section{Background}

Malaria remains a major public health problem which affected about 207 million people and caused an estimated 627,000 deaths in 2012 [1]. In the context of the widespread and increasing occurrence of Plasmodium falciparum resistance against current anti-malarial therapy, new anti-malarial compounds are urgently needed to treat this major endemic disease. In this perspective, it is interesting to note that for many synthetic anti-malarial drugs, $P$. falciparum-resistant isolates were observed one to 12 years after the first use, whereas it was longer for the natural compounds [2]. Indeed, the first reported resistance towards quinine appeared 278 years after its introduction [3]. The use of artemisinin combination therapy

\footnotetext{
* Correspondence: nicolas.taudon@irba.fr

${ }^{2}$ UMR-MD3, Institut de recherche biomédicale des armées, BP73 91223

Brétigny-sur-Orge, France

Full list of author information is available at the end of the article
}

(ACT) as first-line treatment of uncomplicated malaria caused by $P$. falciparum was officially recommended by the WHO in 2006 [4]. Unfortunately, 2,000 years after the use of Artemisia annua in the Chinese Pharmacopoeia to treat fever, the emergence of resistance to artemisinin derivatives was recently reported from Southeast Asia $[5,6]$. Molecules, structurally different from the available antimalarial drugs and targeting innovative and independent metabolism pathways, are particularly needed to prevent the apparition of resistance and to improve care. Drawing from the rich plant biodiversity, new chemical structures may be helpful in the fight against malaria [7].

The ethnopharmacology, based on traditional medicine, offers interesting possibilities in the discovery of new bioactive compounds isolated from the nature. A collaboration between the Cambodian and French (UMR-MD3) faculties allowed inquiries on 28 Cambodian plants used 
in traditional medicine [8]. This work allowed the selection of Stephania rotunda, Brucea javanica, Phyllanthus urinaria and Eurycoma longifolia, among which, S. rotunda (Menispermaceae), a creeping plant growing on calcareous cliffs of Cambodian mountain areas [9], exhibited the most interesting antiplasmodial activity in vitro. Concentrations inhibiting $50 \%$ of parasitic growth $\left(\mathrm{IC}_{50}\right)$ of the dichloromethane and water extracts of $S$. rotunda tuber were below $5 \mu \mathrm{g} / \mathrm{ml}$ on the Plasmodium strain W2 [8]. The fractionation of dichloromethane extracts allowed the isolation of nine alkaloids. The main compound is a bisbenzylisoquinoline, named cepharanthine. This alkaloid has recently been extracted by green chemistry using ultrasound and microwave technologies [10].

Possessing an interesting $\mathrm{IC}_{50}$ measured by flow cytometry $(0.61 \mu \mathrm{M}$ on $\mathrm{W} 2$ strain), the antiplasmodial activity of cepharanthine was tested in mice infected by Plasmodium berghei at a dose of $10 \mathrm{mg} / \mathrm{kg}$ [8]. By intraperitoneal injection and oral administration, this alkaloid decreased the parasitaemia by 47 and 50\%, respectively. Despite the absence of mice sterilization, this molecule is interesting in combination with other anti-malarial drugs. Indeed, cepharanthine possesses a synergistic activity with chloroquine $[8,11]$ but the mechanism of this potentiation is not known currently. Two hypotheses have been proposed to explain this phenomenon: an alteration of the parasite membrane potential [12] or a modulation of P-glycoprotein [13] by cepharanthine.

Previous work showed that cepharanthine seemed to possess a putative mechanism of action different from those of anti-malarial drugs commonly used. Indeed, cepharanthine did not affect the crystallization of haem, unlike chloroquine. The measurement of mitochondrial membrane depolarization after labelling with DiOC6 did not show any effect on the mitochondrial membrane potential by cepharanthine, contrary to atovaquone. The use of ascorbic acid as a potential inhibitor of free radical production did not reveal any activity of free radicals production for cepharanthine, contrary to artemisinin and its derivatives [14].

The work presented here highlights potential plasmodial targets of cepharanthine using both phenotypic and transcriptional approaches.

\section{Methods}

\section{Drug sensitivity assay}

Chloroquine (CQ) and mefloquine (MQ) drugs were purchased from Sigma (St Louis, MO, USA). CQ-resistant/ MQ-susceptible clones FCM2 (Cameroon), W2 (Vietnam), K1 (Thailand), and CQ-susceptible/MQ- resistant 3D7 strain (from NF54 African strain, MR4: Malaria Research and Reference Reagent Resource centre) were used in this study. Parasites were cultivated in type $\mathrm{A}^{+}$human erythrocytes (2\% haematocrit) suspended in RPMI 1640 medium
(Invitrogen, Paisley, UK) supplemented with 10\% human serum (Abcys SA, Paris, France) and buffered with $25 \mathrm{mM}$ HEPES-25 mM $\mathrm{NaHCO}_{3}$ under controlled atmospheric conditions $\left(10 \% \mathrm{O}_{2}, 5 \% \mathrm{CO}_{2}\right.$, and $\left.85 \% \mathrm{~N}_{2}\right)$ at $37^{\circ} \mathrm{C}$ with $95 \%$ humidity. Cultures were synchronized at the ring stage by two successive D-sorbitol $5 \%(\mathrm{~m} / \mathrm{v})$ (Sigma-Aldrich) treatments, which were applied with an interval of four hours; the first to old schizonts in the process of releasing the merozoites and the second to rings obtained from the merozoites release to kill mature schizonts still in the culture [15]. The in vitro assay has been performed once, in triplicate, on all strains simultaneously. Then the $\mathrm{IC}_{50}$ values obtained for positive controls (MQ and CQ) allow the validation of the results by comparison with bibliographic references.

Cepharanthine (>99\%) was extracted from the S. rotunda tuber, according to a percolation method previously validated in the Laboratory of Pharmacognosy and Ethnopharmacology of Aix-Marseille University [16]. The culture of $P$. falciparum strains was performed with the method of Trager and Jensen [17]. Cepharanthine $\mathrm{IC}_{50}$ levels were determinate on the four plasmodial strains, according to Desjardins method [18], using tritied hypoxanthine with a specific activity of $5 \mathrm{mCi} / 5 \mathrm{~mL}$ (Perkin-Elmer, Courtaboeuf, France) to evaluate the parasitic growth. Stock solutions of the test substances were prepared in 5\% DMSO and 95\% methanol with a final concentration of $395.6 \mu \mathrm{M}$. CQ and MQ were used as references. Cepharanthine, CQ and MQ activities were evaluated as a ten serial two-fold dilutions of, respectively, 19.53-20,000, 3.95,000 and 0.39-400 nM. The dilutions of each compound were set in a 96-well plate, in triplicate, and dried overnight. Test solutions were then mixed with a suspension of infected human red blood cells to achieve a final haematocrit of $1.5 \%$ and parasitaemia of $0.8 \%$, and incubated for $48 \mathrm{hr}$ in microtitre plates $\left(200 \mu \mathrm{L}\right.$ final volume). $\mathrm{IC}_{50}$ levels, i e, the drug concentration corresponding to $50 \%$ of the uptake of $\left[{ }^{3} \mathrm{H}\right]$ hypoxanthine by the parasites in drugfree control wells, was determined by non-linear regression analysis of log dose response curves (Riasmart, Packard, Meriden, NJ, USA).

\section{Morphologic characterization of cepharanthine effects}

As described above, 45 hours after a tight synchronization, the parasites were used to study the effect of cepharanthine. The parasite morphology and parasitaemia were evaluated by microscopic observation $(\times 100)$ of thin blood smears stained with RAL ${ }^{\circledR} 555$ (REACTIFS RAL, Martillac, France).

In order to design the transcriptional assay, three studies were performed on different $P$. falciparum cultures with the 3D7 strain. Parasites were incubated with cepharanthine at the concentration corresponding to the $\mathrm{IC}_{50}$ level. Blood smears were frequently prepared to monitor 
the development of parasite life cycle stages. In a first step, cepharanthine was incubated in a continuous fashion on each erythrocyte stage (ring, trophozoite and schizont) until the third merozoite invasion in the control group, for investigation of a dependent-stage effect. Then, cepharanthine was incubated on ring stage and during the equivalent of one $P$. falciparum life cycle ( $45 \mathrm{hr}$ ). After this treatment, cultures were washed three times and blood smears were prepared during a 90-hr period to follow-up a potential parasitic recrudescence. In a last experiment, cepharanthine was added continuously during $144 \mathrm{hr}$ on rings aged of $4 \mathrm{hr}, 10 \mathrm{hr}$ or $16 \mathrm{hr}$. The aim of this assay was to ascertain a potential effect depending on the time of cepharanthine incubation after the merozoite invasion.

\section{Transcriptional analysis}

\section{Parasitic treatment and RNA extraction}

Cultures of $P$. falciparum 3D7 strain were closely synchronized in ring stages by two sequential D-sorbitol $5 \%$ treatments. One life cycle later, re-invasion and synchronization were checked on thin blood smears. Cultures were pooled and divided into 12 samples to constitute four biological replicates for each the three analytic groups: UT8 and UT16 were the controls $8 \mathrm{hr}$ and $16 \mathrm{hr}$ post-merozoite invasion ( $\mathrm{h}$ pmi), respectively; T16 was the group exposed to a 8 -hr period of cepharanthine incubation started at $8 \mathrm{hr}$ pmi (Figure 1). Cepharanthine was used at the concentration corresponding to the $\mathrm{IC}_{90}$ estimated graphically from the curve modeled for $\mathrm{IC}_{50}$ calculation on the 3D7 strain. As the duration of the ring stage period was $20 \mathrm{hr}$ pmi, this time-window prevented invasion events and enabled a selective assay. RNA was extracted from erythrocyte pellets blocked by TRIZOL ${ }^{\mathrm{TM}}$ reagent according to manufacturer's recommendations (Invitrogen, Carlsbad, CA, USA), and treated with DNase (DNAfree ${ }^{\mathrm{Tx}}$, Ambion, Foster City, CA,
USA). RNA samples were checked for integrity using the NanoDrop ND-1000 (Labtech, Palaiseau, France) and qualified using a 2100 Bioanalyzer RNA Nano Chip (Agilent Technologies, Santa Clara, CA, USA) according to the manufacturer's protocol. No trace of DNA contamination was detected by Bioanalyzer analysis.

\section{Microarray assay and analysis}

A reference RNA was obtained pooling an equal amount of RNA of each sample (UT8, UT16 and T16) to normalize the data arising from the microarray experiment [19]. Microarrays were performed using the Agilent protocol version 6.5 (Low Input Quick Amp Labeling). Cyanine-3 CTP $(\mathrm{Cy}-3)$ fluorescent dye was used to label the samples and Cy-5 fluorescent dye to label the reference (RNA pool). The labelled cRNA were hybridized on the P. falciparum $4 \times 44 \mathrm{k}$ Agilent custom microarrays developed in the parasitology laboratory of Army Biomedical Research Institute (Marseille). On these microarrays, 9,722 probes were spotted, corresponding to 5,144 genes. Microarrays were scanned using an Agilent Scanner (G2505B). Data were extracted and normalized from the scanned images using the Agilent Feature Extraction software (ver 9.5.3.1). The annotation of the microarray was updated using the PlasmoDB 9.0 annotations [20] and the Malaria Parasite Metabolic Pathways [21]. The version 9.0 of PlasmoDB introduced a new name code for each $P$. falciparum gene and updated the Gene Ontology (GO) annotation. Two-way ANOVA analyses (time-treatment) were performed with a FDR correction and a p-value of 0.05 on normalized data using the Genespring GX software (ver 12.0). The significant, differentially expressed probes were filtered using a fold change (FC) above two between UT8, UT16 and T16. Genespring allowed the computation of a hierarchical clustering (Euclidian metric, centroid method) and GO
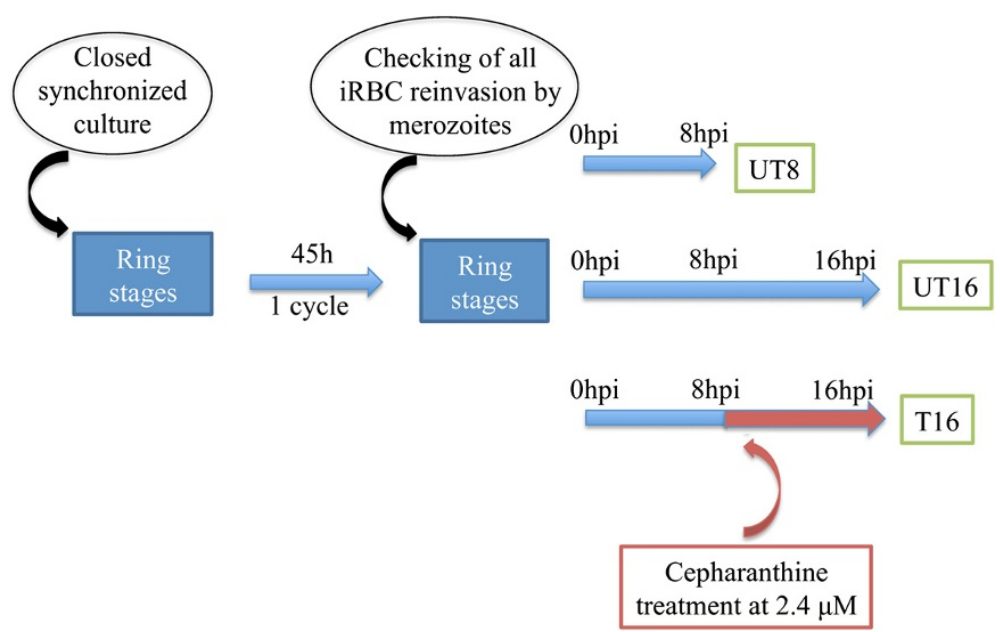

Figure 1 Design of the transcriptional assay. UT8 = untreated samples $8 \mathrm{hr}$ post-invasion by merozoite ( $\mathrm{h}$ pmi); UT16=untreated samples $16 \mathrm{~h}$ pmi; T16 = samples treated by cepharanthine at $8 \mathrm{~h}$ pmi during eight hours; iRBC = infected red blood cells. 
analyses. The significantly over-represented GOs were input in QuickGO [22] in order to produce an Ancestral Chart, representing these GOs with their relationships.

The pathway analyses were performed using the statistical software $\mathrm{R}$ [23] and the Malaria Parasite Metabolic Pathways database [21] as reference. The over-representations of pathways were determined by Fisher exact t-tests with a p-value threshold of 0.05 . Then, the significant pathways are grouped by functions according to the Malaria Parasite Metabolic Pathways database.

\section{Microarray validation by real-time quantitative polymer- ase chain reaction}

The real-time quantitative polymerase chain reaction (qRT-PCR) was performed on an Applied Biosystems 7900 Fast Real-Time PCR system (Carlsbad, California, USA). Primers were designed using the Applied Biosystems software Primer Express (ver 2.0.0). In order to avoid genomic DNA contamination, the following rules have been used for the primers. The primers were designed close to the 3 ' end of the genes to take into account the reverse transcriptase step. If possible, we chose primers spanning intron. Due to the particular $P$. falciparum nucleotide composition, the size of the primers were selected between 18 and $35 \mathrm{nt}$, with a Tm between 56 and $62^{\circ} \mathrm{C}$. The primers were previously tested at two different concentrations $(0.5$ and $0.9 \mu \mathrm{M})$ to select the most efficient one. For each primer, the used concentration was different (see Additional file 1). Efficiency was calculated by the following formula:

$$
\mathrm{E}=\left(10^{(-1 / \text { slope })}-1\right) \times 100
$$

Efficiency must be high (superior to 0.95) and constant among samples.

One microgram of the DNase-treated total RNA was reverse-transcribed with the High-Capacity cDNA Archive kit (Applied Biosystems, Carlsbad, CA, USA). The expression of 12 genes was evaluated on each cDNA sample (see Additional file 1). PCR amplifications were carried out using $12.5 \mu \mathrm{L} \mathrm{SYBR}{ }^{\circ}$ Green PCR Master Mix (2X) (Power SYBR ${ }^{\circ}$ Green, Applied Biosystems, Carlsbad, CA, USA), $2.5 \mu \mathrm{L}$ of each primer and $5 \mu \mathrm{L}$ of template DNA in a final volume of $25 \mu \mathrm{L}$. The thermal cycling conditions were $95^{\circ} \mathrm{C}$ for $10 \mathrm{~min}$, and 40 cycles of $95^{\circ} \mathrm{C}$ for $15 \mathrm{sec}$, then $60^{\circ} \mathrm{C}$ for $60 \mathrm{sec}$. For each gene, a no template control was used (water). The fluorescence acquisition was performed at the end of each extension step. The measurements were performed in triplicate for all the samples and genes. The $2^{-\Delta \Delta C t}$ formula was applied to normalize the detected fluorescent signal with endogenous reference Plasmodium ribosomal small subunit $18 \mathrm{~s}$ rRNA and to compare each sample with the controls.

$$
\begin{aligned}
& \Delta \mathrm{Ct}=\mathrm{Ct}_{\text {sample }}-\mathrm{Ct}_{18 \text { SrRNA }} \\
& \Delta \Delta \mathrm{Ct}=\Delta \mathrm{Ct}_{\text {treated }}-\Delta \mathrm{Ct}_{\text {control }}
\end{aligned}
$$

\section{Results}

\section{Drug sensitivity assay}

The $\mathrm{IC}_{50}$ values of cepharanthine, $\mathrm{CQ}$ and $\mathrm{MQ}$ were respectively between 927 and 3,059 nM, 21.2 and $738 \mathrm{nM}$, 14.3 and $67.0 \mathrm{nM}$ depending on the P. falciparum strain used (Table 1). The levels of activity obtained for CQ and MQ were in accordance with the results found in the literature $[24,25]$. The levels of relative sensitivity of cepharanthine $v s \mathrm{CQ}$ and MQ were different regarding strains. $\mathrm{IC}_{50}$ ratios of cepharanthine/CQ and cepharanthine/MQ were between (1.6-107), and (34.0-126), respectively.

\section{Morphologic characterization of cepharanthine effects}

Morphological assays were performed on close synchronized cultures to highlight a potential effect of cepharanthine depending of the Plasmodium stage. Indeed, such referrals were required beforehand to design a relevant transcriptional assay. Parasites were incubated with cepharanthine at the concentration corresponding to the $\mathrm{IC}_{50}$ level on the 3D7 stain.

In a first step, cepharanthine was incubated in a continuous fashion on each erythrocyte stage (ring, trophozoite and schizont). Ring stages could not differentiate into trophozoites. While at trophozoite and schizont stages a decrease in parasitaemia was observed. The surviving parasites progressed in their life cycle until the next ring stage, and then differentiation was definitively blocked.

After incubation on ring stages during a 45-hr period, parasites resumed their growth according a normal life cycle about $48 \mathrm{hr}$ after removal of the drug pressure. Thus, cepharanthine could have a parasitostatic rather than a parasitocidal effect.

Effect of cepharanthine inside the ring stage was investigated by incubating the drug at 4, 10 and $16 \mathrm{hr}$ pmi during a 144-hr period. As described above, parasitical growth was blocked whatever the delay for incubation. The parasitaemia was not positive at the end of the observation period.

Based on all these morphological observations, a microarray approach targeting effect of cepharanthine on Plasmodium ring stage has been designed.

\section{Transcriptional assay}

The use of microarrays allowed the study of cepharanthine impact on the entire Plasmodium transcriptome simultaneously and underlined the probable pathways 
Table 1 I $C_{50}$ of cepharanthine against four Plasmodium falciparum strains

\begin{tabular}{lllll}
\hline Compound/strain & FCM29 & W2 & 3D7 & K1 \\
\hline Cepharanthine & $3059(12.3 \%)$ & $927(6.5 \%)$ & $2276(15.8 \%)$ & $1803(17.5 \%)$ \\
Chloroquine (CQ) & $738(7.2 \%)$ & $572(19.5 \%)$ & $21.2(13.7 \%)$ & $164(35.1 \%)$ \\
Mefloquine (MQ) & $24.5(10.1 \%)$ & $26.5(9.2 \%)$ & $67.0(5.9 \%)$ & $14.3(28.0 \%)$ \\
$I C_{50}$ ratio (Cepharanthine/CQ) & 4.1 & 1.6 & 107 & 11.0 \\
$I C_{50}$ ratio (Cepharanthine/MQ) & 125 & 35.0 & 34.0 & 126 \\
$I C_{50}$ ratio (CQ/MQ) & 30.1 & 21.6 & 0.3 & 11.5 \\
\hline
\end{tabular}

The first three rows are $\mathrm{IC}_{50}$ (arithmetic mean in $\mathrm{nM}, \mathrm{RSD} \%$ ) of three drugs on four Plasmodium falciparum strains. These values have been computed using triplicates $(n=3)$. The last three rows are the $I_{50}$ ratio between every pair of the tested drugs.

impacted by this compound. The sampling times for the design of transcriptional analysis were determined as 8 and $16 \mathrm{~h} \mathrm{pmi}$ and the concentration of cepharanthine was $5 \mu \mathrm{M}$. Applying a two-way ANOVA analysis and a FC threshold of two, 1,141 probes corresponding to 781 genes showed a significant variation of expression (see Additional file 2). In PlasmoDB 9.0, 44.7\% of these probes have been annotated as unknown function. A hierarchical clustering showed a wide proximity between UT8 and T16 (Figure 2). The probes possessing the similar expression profiles were grouped in the same cluster. Two groups of probes were differentially expressed between UT8 and T16: probes under- (in blue) or over-expressed (in red) by cepharanthine. The comparison of probes expression between UT16 and T16 showed no similarity, indicating that the entire transcriptome was affected by the cepharanthine treatment.

The most important variations of expression were observed for six genes families (see Additional file 2). The FCs obtained from surface-associated, interspersed gene (SURFIN) were between -5 and +9 (UT16 vs T16 for PF13_0074 and UT8 vs UT16 for PFA0655w, respectively). For STEVOR, these values were ranged between -17 and +62 (UT8 vs UT16 and UT16 vs T16, respectively for PF10_0395). The RIFIN FCs were contained between -13 and +27 (UT8 vs UT16 and UT16 vs T16, respectively for PFD0055w). The FCs of protein kinases were between -4.5 and +5.5 (UT8 vs UT16 for, respectively MAL13P1.185 and PF14_0264). The FC values of Plasmodium-exported proteins (PHIST and hyp) were ranged between -19.5 and +11 (UT8 vs UT16 for, respectively PFA0700c and MAL8P1.160). The P. falciparum two-transmembrane Maurer's cleft protein (Pfmc-2TM) FCs were between -16 and +24.6 (UT8 vs UT16 for PFA0065w and UT16 vs T16 for PF11_0014, respectively).

\section{Gene Ontology and pathways analysis}

The GO analysis has been performed on the differentially expressed genes. Sixty GOs terms are significantly over-represented (Fisher exact t-test, p-value $<0.05$ ). These GOs involved particularly 'cellular components' with Maurer's clefts and apicoplast; 'biological process' with antigenic variation, glyconeogenesis and mitochondria. Only three significant GOs, involving amine and lipid binding, were found in the 'molecular function' group (see Additional file 3).

Pathways analysis showed few differences between UT8 and T16. The most impacted function between these two experimental conditions seemed to be invasion and motility, containing two pathways under-expressed by cepharanthine (the functional annotation of merozoite invasion-related proteins and the subcellular localization of proteins involved in invasion) (see Additional file 4). A Fisher's exact test right showed that 20 pathways were significantly modified by cepharanthine ( $\mathrm{p}$-value $<0.1$ ) (see Additional file 5). According to the functional classification of genes obtained (Figure 3), cyto-adherence and rosetting, included in cell-cell interaction group, are the most significant pathways $\left(\mathrm{p}\right.$-value $=2.49 \times 10^{-13}$ and $1.54 \times 10^{-11}$, respectively). Cyto-adherence corresponds to the capacity of parasitized erythrocytes to adhere to endothelial cells, whereas rosetting corresponds to the ability of parasitized erythrocytes to adhere to uninfected erythrocytes. These two properties are responsible for the sequestration of infected red blood cells, mainly in capillaries of the deep microvasculature. The organellar functions, including mitochondrion and apicoplast, are significant pathways in this analysis. These two organelles were also cited by the $\mathrm{GO}$ analysis and are important for the parasite [26]. The histograms confirmed that a treatment with cepharanthine decreases cytoadherence, Maurer's clefts, apicoplast and S-glutathionylated protein pathways compared to initial and final controls (see Additional file 4).

\section{Microarray validation by qRT-PCR}

The differentially expressed transcripts were validated by qRT-PCR. The variation of expression of 12 transcripts was evaluated and compared with the microarray results. A good concordance was observed for all transcripts and conditions, as shown in Figure 4 (comparing UT16 and T16), Figure 5 (comparing UT8 and T16); and Figure 6 (comparing UT8 and UT16). The Spearman rank correlation method gives p-value of $8.67 \times 10^{-6}, 2.04 \times 10^{-6}$ and $3.13 \times$ $10^{-7}$ for the comparisons in Figures 4, 5 and 6, respectively. 
available anti-malarial drugs [14]. This assumption was not contradicted by the evaluation of $\mathrm{IC}_{50}$ levels on strains that have various sensitivities to anti-malarial drugs. Indeed, assays performed on 3D7 strain (CQ-sensitive/MQresistant) and on the Asian W2, FCM29 and K1 strains (CQ-resistant/MQ-sensitive) showed that the $\mathrm{IC}_{50}$ ratios of cepharanthine on $\mathrm{CQ}$ and $\mathrm{MQ}$ moved depending on the stains. For example, the susceptibility of W2 to cepharanthine was higher than $\mathrm{K} 1$ whereas the opposite was observed with both $C Q$ and $M Q$. Furthermore, morphologic characterization of cepharanthine impact on the life cycle of Plasmodium showed a particular effect depending on the parasite stage. Indeed, at any time of the parasite life cycle, when cepharanthine was incubated with a constant drug pressure, Plasmodium was blocked at the ring stages and parasitaemia followed down to $0 \%$ after a 96- to 144hr period. When the drug was removed, the life cycle of the parasite returned to normal. This stage effect of cepharanthine is highlighted for the first time. This specificity has enabled the design of a microarray assay at the ring stage and on closely synchronized cultures.

At the transcriptional level, a tight synchronization of parasites was performed within a four-hour timewindow, allowing good accuracy of microarray results and reflecting the quality of the study. The transcriptional assay was performed during a short time of eight hours and on a relatively stable parasitic stage. Indeed, the ring stage corresponded to the beginning of the Plasmodium life cycle in which gene expression is slightly modified, few metabolic pathways being established. Despite this, high variations of gene expression were observed between the three conditions studied. This transcriptional study highlights the set of genes whose expression is directly and indirectly disrupted by cepharanthine. Transcriptional analysis performed on ring stage confirmed that the parasitic blockage, microscopically observed, might be related to the metabolism pathways.

The limitations of this study are mainly due to Plasmodium model. The quickness of Plasmodium cycle and the constancy of the parasitic evolution explain the choice of working on short time. The design of the transcriptional experiment performed on ring stages was not easy, involving a large amount of synchronized ring stages and a sufficient incubation time of cepharanthine. So, time points have been chosen to be far from the invasion by merozoites and the transformation into trophozoite stages.

Asahi et al. studied factors controlling intra-erythrocyte development of P. falciparum [27]. They used various chemically defined mediums and after transcriptome profiling, they found 26 transcripts predicted to be associated with the schizogony stunting. Two of their over-expressed transcripts were also significantly upregulated in the study presented here (CSP-TRAP PFC0640w and MYND finger protein PFF0350w). The upregulation of these two genes

\section{Discussion}

A previous study had suggested that cepharanthine could have an antiplasmodial activity differing from those of 


\title{
Functional classification of genes
}

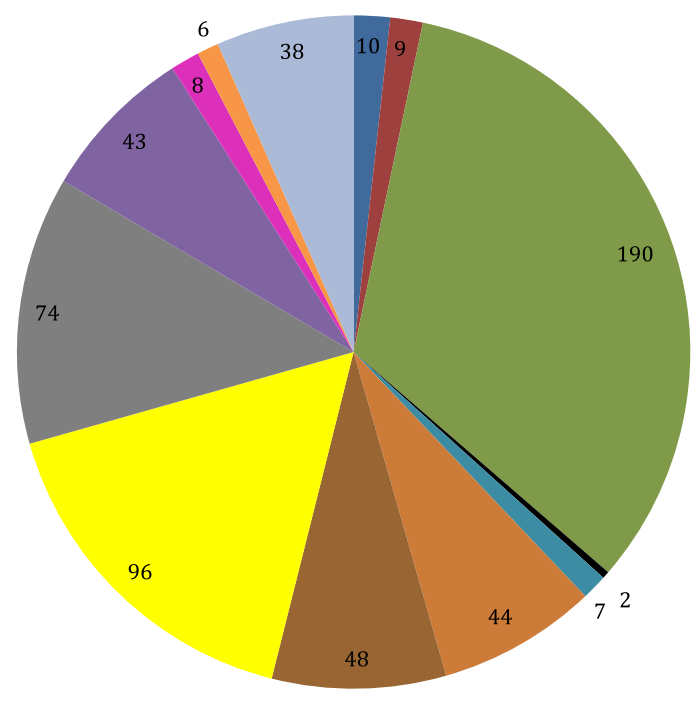

\author{
- Amino Acid \\ - Carbohydrate \\ Cell-Cell interaction \\ - Cofactors \& other substances \\ Haemoglobin digestion \\ Invasion \& motility \\ - Lipids \\ Organellar function \\ - Post translational modifications \\ - Protein traffic \\ - Redox metabolism \\ Transcription \\ - Transport
}

Figure 3 Functional classification of genes with expression modified by cepharanthine. The number of genes in each category is indicated in the outer circle (e.g. 21. Malaria Parasite Metabolic Pathways [http://priweb.cc.huji.ac.il/malaria/]).

could be responsible of the blockage of parasites into ring stage by cepharanthine.

A parasitostatic effect on ring stage was also observed after a treatment with a natural triterpene, named limonene [28]. The freezing of parasite progression from the ring to the trophozoite stage by the limonene could involve a decrease of isoprenylation of proteins as well as rhoptry-associated proteins (RAP). In the study described here, the isoprenylation metabolic pathway was significantly decreased by cepharanthine. This biochemical

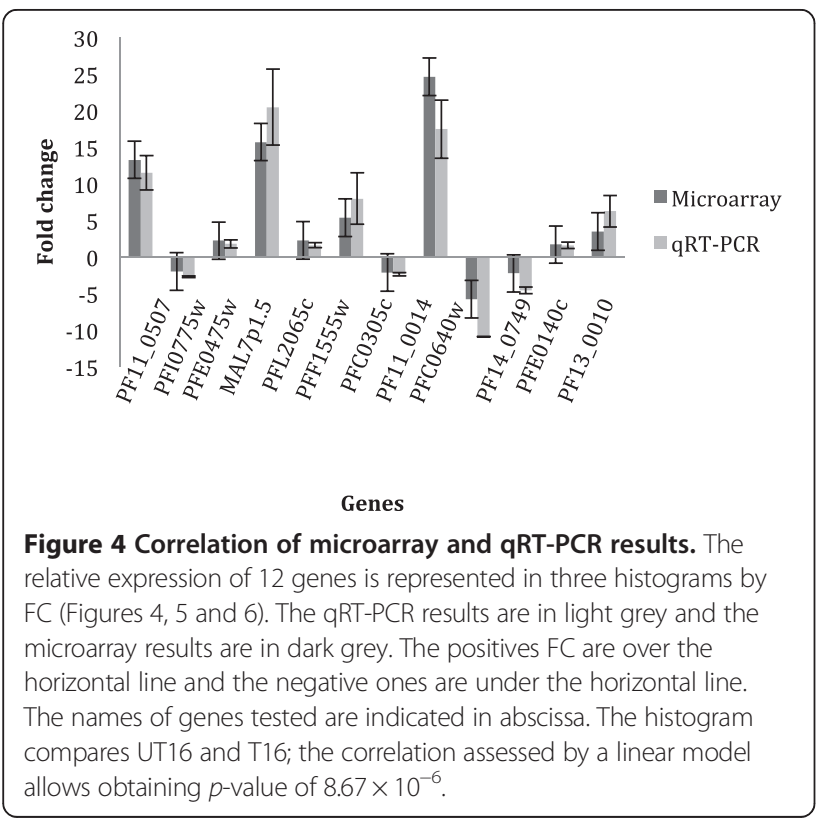

pathway localized in the apicoplast of the parasite is an interesting target because of its absence in the human host. Moreover, the isoprenoid precursor synthesis is essential for the parasite survival $[28,29]$.

At the genomic level, four enzymes of the glycolysis (glucose-6-phosphate isomerase PF14_0341, triose phosphate isomerase PF14_0378, phosphoglycerate mutase PF11_0208 and enolase PF10_0155) and one enzyme of the gluconeogenesis (phosphoenolpyruvate carboxykinase PF13_0234) were down-regulated by cepharanthine and could be responsible for this parasitic blockage. For example, the glucose-6-phosphate isomerase interferes with

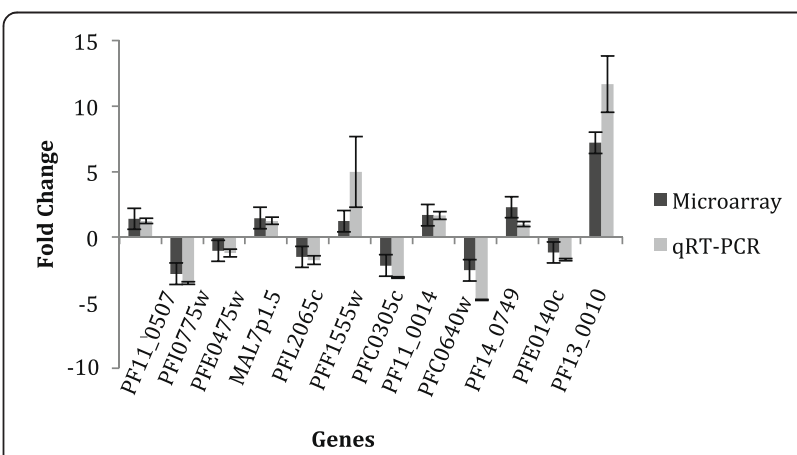

Figure $\mathbf{5}$ Correlation of microarray and qRT-PCR results. The relative expression of 12 genes is represented in these three histograms by FC (Figures 4, 5 and 6). The qRT-PCR results are in light grey and the microarray results are in dark grey. The positives FC are over the horizontal line and the negative ones are under the horizontal line. The names of genes tested are indicated in abscissa. The histogram compares UT8 and T16; the correlation assessed by a linear model allows obtaining $p$-value of $2.04 \times 10^{-6}$ 


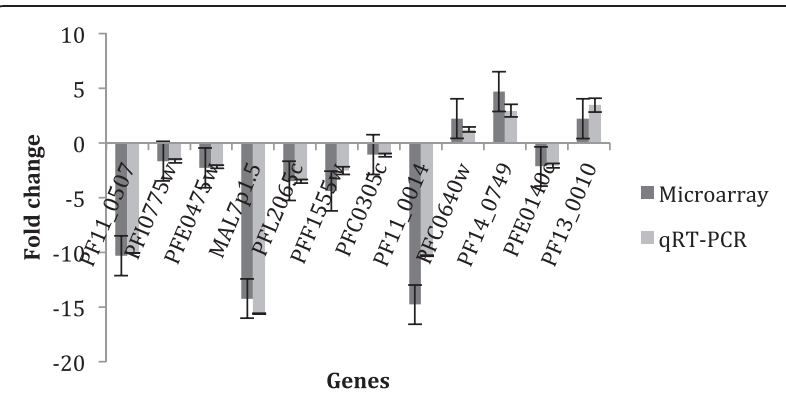

Figure 6 Correlation of microarray and qRT-PCR results. The relative expression of 12 genes is represented in these three histograms by FC (Figures 4, 5 and 6). The qRT-PCR results are in light grey and the microarray results are in dark grey. The positives FC are over the horizontal line and the negative ones are under the horizontal line. The names of genes tested are indicated in abscissa. The histogram compares UT8 and UT16; the correlation assessed by a linear model allows obtaining $p$-value of $3.13 \times 10^{-7}$.

the second step of the glycolysis corresponding to the conversion of glucose 6-phosphate (G6P) in fructose 6phosphate (F6P) [30]. In the GO analysis, the gluconeogenesis was enriched and in the metabolic pathway, the glycolysis was significantly impacted by cepharanthine. According to Bozdech et al., genes of the glycolysis are induced during the ring and early trophozoite stages [31]. A downregulation of genes of this metabolic pathway could be responsible for inhibiting the passage of the ring stage to trophozoite stage.

Among the gene families whose expression is downregulated by cepharanthine, some were quoted as essential for parasite survival. The carbon catabolite repressor protein 4-associated factor 1 (CAF-1) exerts a regulation mainly on the red blood cell invasion by merozoite [32]. The cyclin-dependent kinase cdc2 possesses a regulatory function on the cell cycle evolution in Plasmodium $[33,34]$. The caseinolytic proteases (ClpB) are chaperones located in the apicoplast and involved in the cellular homeostasis [35]. The heat shock proteins (HSP) 40, 70, 90 are among chaperones playing an important role in the cellular processes of the parasite survival and pathogenicity $[26,36,37]$. The SURFIN corresponds to an antigen transported to the red blood cell surface by Maurer's clefts and located at the merozoite apex. This antigen seems fundamental for the merozoite invasion and parasite survival [38-41]. Others were proposed as potential anti-malarial targets. The acyl-CoA binding proteins (ACBP1 and ACBP2) are involved in the de novo apicoplast fatty acid biosynthesis [42]. The aquaglyceroporins are responsible of the urea and glycerol transport [43]. The 4-(cytidine 5'-diphospho)-2-C-methyl-D-erythritol kinases (CMK) play a catalytic role in the biosynthesis of isopentenyl pyrophosphate [44]. Two antigens were proposed as malaria vaccine candidates. The glycosylphosphatidylinositol (GPI)-anchored proteins are responsible of the membranous protein binding and merozoite invasion [45]. The rhoptry-associated proteins (RAP) are immunogenic and involved in the merozoite invasion too $[46,47]$. As both protein targets are well characterized, a Western blot experiment could be performed to confirm the results obtained with the transcriptional assay.

The pathways analysis showed that few pathways are upregulated by cepharanthine. Thus, for a better knowledge of the cepharanthine mechanism and its possible targets, the study focused on genes involved in parasite survival and virulence, downregulated by cepharanthine. The genes' coding for proteins exported to the host cell by Maurer's clefts, named the exportome [26], are generally downregulated by anti-malarials. These clefts are membranous structures involved in the export of parasitic proteins to the erythrocyte membrane [38] and are widely affected by cepharanthine treatment (GO over-represented with a $\mathrm{p}$-value of $10^{-25}$ ). This expression modulation is observed for structural proteins of Maurer clefts (Pfmc2TM [26,48-50] and antigen 332) and proteins exported by Maurer's clefts (ring-infected surface antigen (RESA)-like with PHIST and Dna J domain [26], Plasmodium helical interspersed subtelomeric (PHIST) a, b and c [26], kinase named FIKK [26,51-54]). Currently, there are no available anti-malarial drugs acting directly on the transport mediated by Maurer's clefts. It has been shown that the Maurer's clefts decreased in number but not in function, when treated with artesunate, quinine and piperaquine [55]. However, this observation does not represent the main mechanism of action of these three drugs. It will be necessary to study the functional modifications of these structures before and after treatment by cepharanthine, with additional methods as imaging techniques, to explain the relationship between cepharanthine and Maurer's clefts.

The GOs of host cell plasma membrane and antigenic variations, containing mainly genes coding for PfEMP1, RIFIN and STEVOR, were significantly under-represented in this study $\left(10^{-30}<\mathrm{p}<10^{-4}\right)$. PfEMP1 is addressed to the host erythrocytes by Maurer's clefts and is responsible of cyto-adherence inducing cerebral malaria [56]. Rifin and stevor contribute to the antigenic variation of Plasmodium conferring its adaptability towards all the antiplasmodial treatments. The downregulation of these three kinds of genes by cepharanthine seems interesting for the inhibition of Plasmodium virulence. Furthermore, cepharanthine seems to inhibit genes related to pathways involving mitochondrion $\left(\mathrm{p}=1.5 \times 10^{-4}\right)$ and apicoplast $\left(\mathrm{p}=8.4 \times 10^{-5}\right)$. Proteomic studies showed that these organelles seem to be targeted by doxycycline [57]. Moreover, targeting mitochondrion electron transport, atovaquone induced a static state on the ring stages [58]. The parasitostatic effect observed with cepharanthine treatment could be due to its activity on mitochondrion but also on apicoplast. Indeed, a "delay death" has been observed with drugs inhibiting 
apicoplast as tetracycline and fosmidomycin [59]. The mechanism involved in this phenomenon is not yet elucidated but it is traduced by a blockage of parasitic growth after the reinvasion of erythrocytes [29]. This property has also been observed with cepharanthine that induced a blockage of ring stages during the second parasitic cycle. Moreover, being absent in humans, the apicoplast is a specific target. The compounds acting on this organelle could induce good safety in humans. So, it would be interesting to study the effect of cepharanthine on this organelle with further complementary and specific experiments to a better understanding and characterization of this inhibition of isoprenoid precursor biosynthesis.

In the goal to identify the real targets of cepharanthine, the potential targets underlined at the transcriptomic level in this work have to be confirmed at the proteomic level. Moreover the use of imagery technics could be interesting to check the activity of cepharanthine on some targets as Maurer clefts and mitochondrion. Elsewhere the activity of cepharanthine on cytoadherence could be evaluated in vitro and in vivo using the Palo-Alto (FUP)1 P. falciparum strain [60].

Previous pharmacokinetic studies performed in mouse [61], beagle dog and human [62] showed a quite long elimination half-life for cepharanthine that could be a potential candidate in combination with faster-acting antimalarials in the treatment of multidrug-resistant falciparum malaria in seriously ill patients (ACT combination for example) [11].

\section{Conclusions}

In this work, the bisbenzylisoquinoline, called cepharanthine, exerted an antiplasmodial activity against four strains of $P$. falciparum. There is no bisbenzylisoquinoline currently used in the treatment of malaria, so, in light of the development of resistance against standard antimalarials and the search for new drugs with a novel mechanism of action, cepharanthine could be a potential drug lead. Phenotypic and transcriptional assays showed for the first time a blocking effect of cepharanthine into ring stage. This parasitostatic effect seems to involve the isoprenoid and glycolysis metabolic pathways probably by the inhibition of various enzymes of these pathways. The gene expression profiling by microarray showed that cepharanthine could interfere with several important functions for Plasmodium survival and virulence as the mitochondrion, apicoplast, cytoadherence antigenic variation and Maurer's clefts. Further studies directly targeting the genes of interest are needed to confirm the potential targets. These original potential targets could allow for the use of cepharanthine in a new drug combination. The implementation of complementary approaches as proteomic or metabolomics studies for example, is necessary to confirm results obtained at the genomic level.

\section{Additional files}

\begin{abstract}
Additional file 1: Efficiencies and sequences of the forward (F) and reverse (R) primers. For each probe, the new and old accession numbers, the name and acid nucleic sequences are given. $R^{2}$, slope and efficiency were calculated to choose the best concentration to use for the qRT-PCR experiment.
\end{abstract}

Additional file 2: Fold-change of probes comparing the three conditions UT8, UT16 and T16.

Additional file 3: Hierarchical classification of Gene Ontology terms enriched during the transcriptomic experiment.

Additional file 4: Differences of expression in pathways due to cepharanthine. These three histograms compare the expression of pathways by pairs of experimental conditions. Horizontal bars lengths correspond to the number of genes up-regulated (red) and down-regulated (green) from each metabolic pathway. FC= fold change, UT = untreated, $T=$ treated.

Additional file 5: Metabolic pathways modified by cepharanthine. Over-represented pathways were grouped into functions according to the Malaria Parasite Metabolic Pathways database. P-values were obtained by Fisher exact t-tests. The threshold of significance is 0.05 .

\section{Abbreviations}

ACBP: Acyl-CoA binding protein; ANOVA: Analysis of variance; CAF-1: Carbon catabolite repressor protein 4-associated factor 1; ClpB: Caseinolytic protease; CQ: Chloroquine; FC: Fold change; F6P: Fructose 6-phosphate; G6P: Glucose 6-phosphate; GO: Gene ontology; GPI: Glycosylphosphatidylinositol; HSP: Heat shock protein; $I_{50}$ : Concentration inhibiting $50 \%$ of parasitic growth; MQ: Mefloquine; pmi: Merozoite post invasion; PfEMP1: Plasmodium falciparum erythrocyte membrane protein-1; Pfmc-2TM: Plasmodium falciparum Maurer's clefts two-transmembrane protein; PHIST: Plasmodium helical interspersed subtelomeric protein; qRT-PCR: Quantitative real time polymerase chain reaction; RAP: Rhoptry-associated proteins; RESA: Ring-infected surface antigen; RSD: Relative standard deviation; SURFIN: Surface associated interspersed gene; T16: Samples treated by cepharanthine; UT8: Untreated controls arrested $8 \mathrm{hr}$ pmi by merozoites; UT16: Untreated controls arrested 16 hr pmi by merozoites.

\section{Competing interests}

The authors declare that they have no competing interests.

\section{Authors' contributions}

EO, DP, CC, and NT designed and coordinated the study. CD and $C T$ performed the transcriptomic assay. CD, CC, JD, and NT analyzed the transcriptomic data and wrote the manuscript. AP and CD performed the cultivation of Plasmodium strains and the qRT-PCR experiment. BB provided cepharanthine for the study. All the authors read and approved the final manuscript.

\section{Acknowledgements}

This study was supported by the Service de Santé des Armées (SSA) and the Direction Générale de l'Armement (DGA).

\section{Author details}

1UMR-MD3, Institut de recherche biomédicale des armées, Faculté de Pharmacie, Aix-Marseille Université, 27 Bd Jean Moulin CS30064 13385 Marseille cedex 5, Marseille, France. ${ }^{2}$ UMR-MD3, Institut de recherche biomédicale des armées, BP73 91223 Brétigny-sur-Orge, France.

${ }^{3}$ Trypanosome Cell Biology Unit, CNRS URA2581 and Parasitology Department, Institut Pasteur 25 rue du Docteur Roux, 75015 Paris, France. ${ }^{4}$ Département d'Infectiologie de Terrain, Unité de Parasitologie, Institut de Recherche Biomédicale des Armées, Marseille, France. ${ }^{5}$ UMR-MD3, Laboratoire de Pharmacognosie et Ethnopharmacologie, Faculté de Pharmacie, Aix-Marseille Université, 27 Bd Jean Moulin 13385 Marseille Cedex 5, Marseille, France.

Received: 1 May 2014 Accepted: 7 August 2014

Published: 22 August 2014 


\section{References}

1. WHO: World Malaria Report. Geneva: World Health Organization; 2013.

2. Pradines B, Dormoi J, Briolant S, Bogreau H, Rogier C: La résistances aux antipaludiques. Revue Francophone des Laboratoires 2010, 422:51-62.

3. Ekland EH, Fidock DA: In vitro evaluations of antimalarial drugs and their relevance to clinical outcomes. Int J Parasitol 2008, 38:743-747.

4. WHO: Guidelines For The Treatment Of Malaria. Geneva: World Health Organization; 2006

5. Dondorp AM, Nosten F, Yi P, Das D, Phyo AP, Tarning J, Lwin KM, Ariey F, Hanpithakpong W, Lee SJ, Ringwald P, Silamut K, Imwong M, Chotivanich K, Lim P, Herdman T, An SS, Yeung S, Singhasivanon P, Day NP, Lindegardh N, Socheat D, White NJ: Artemisinin resistance in Plasmodium falciparum malaria. N Engl J Med 2009, 361:455-467.

6. Cheeseman IH, Miller BA, Nair S, Nkhoma S, Tan A, Tan JC, Al Saai S, Phyo AP, Moo CL, Lwin KM, McGready R, Ashley E, Imwong M, Stepniewska K, Yi P, Dondorp AM, Mayxay M, Newton PN, White NJ, Nosten F, Ferdig MT, Anderson TJ: A major genome region underlying artemisinin resistance in malaria. Science 2012, 336:79-82

7. Kaur K, Jain M, Kaur T, Jain R: Antimalarials from nature. Bioorg Med Chem 2009, 17:3229-3256.

8. Chea A, Hout S, Bun SS, Tabatadze N, Gasquet M, Azas N, Elias R, Balansard G: Antimalarial activity of alkaloids isolated from Stephania rotunda. J Ethnopharmacol 2007, 112:132-137.

9. Desgrouas C, Taudon N, Bun SS, Baghdikian B, Bory S, Parzy D, Ollivier E: Ethnobotany, phytochemistry and pharmacology of Stephania rotunda Lour. J Ethnopharmacol 2014, 154:537-563.

10. Desgrouas C, Baghdikian B, Mabrouki F, Bory S, Taudon N, Parzy D, Ollivier E: Rapid and green extraction, assisted by microwave and ultrasound of cepharanthine from Stephania rotunda Lour. Separation Purification Technologies 2014, 123:9-14.

11. Desgrouas C, Dormoi J, Chapus C, Ollivier E, Parzy D, Taudon N: In vitro and in vivo combination of cepharanthine with anti-malarial drugs. Malar J 2014, 13:90

12. Haruki K, Bray PG, Ono M, Ward SA: Potent enhancement of the sensitivity of Plasmodium falciparum to chloroquine by the bisbenzylisoquinoline alkaloid cepharanthin. Antimicrob Agents Chemother 2000, 44:2706-2708.

13. Tamez PA, Lantvit D, Lim E, Pezzuto JM: Chemosensitizing action of cepharanthine against drug-resistant human malaria, Plasmodium falciparum. J Ethnopharmacol 2005, 98:137-142.

14. Chea: Ethnopharmacologie de plantes traditionnellement utilisées au Cambodge dans le traitement de la malaria et des maladies infectieuses: application à trois plantes antipaludiques Erioglossum edule Blume [Lepisanthes rubiginosa (Roxb.) Leenh.], Stephania rotunda Lour., Vernonia cinerea Less. Aix-Marseille II: Pharmacognosie; 2006.

15. Lambros C, Vanderberg JP: Synchronization of Plasmodium falciparum erythrocytic stages in culture. J Parasitol 1979, 65:418-420

16. Bory S, Bun S, Baghdikian B, Mabrouki F, Cheng S, Elias R, Bune H, Ollivier E: Simultaneous HPLC determination of three bioactive alkaloids in the Asian medicinal plant Stephania rotunda. Nat Prod Commun 2010, 5:877-882.

17. Trager W, Jensen JB: Human malaria parasites in continuous culture. Science 1976, 193:673-675.

18. Desjardins RE, Canfield CJ, Haynes JD, Chulay JD: Quantitative assessment of antimalarial activity in vitro by a semiautomated microdilution technique. Antimicrob Agents Chemother 1979, 16:710-718

19. Becker JV, Van Der Merwe MM, Van Brummelen AC, Pillay P, Crampton BG, Mmutlane EM, Parkinson C, Van Heerden FR, Crouch NR, Smith PJ, Mancama DT, Maharaj VJ: In vitro anti-plasmodial activity of Dicoma anomala subsp. gerrardii (Asteraceae): identification of its main active constituent, structure-activity relationship studies and gene expression profiling. Malar J 2011, 10:295.

20. Das B, Tandon V, Lyndem LM, Gray Al, Ferro VA: Phytochemicals from Flemingia vestita (Fabaceae) and Stephania glabra (Menispermeaceae) alter cGMP concentration in the cestode Raillietina echinobothrida. Comparative Biochem Physiol Part C Toxicol Pharmacol 2009, 149:397-403.

21. Malaria Parasite Metabolic Pathways. http://mpmp.huji.acil.

22. Gbolade A, Adeyemi A: Investigation of in vitro anthelmintic activities of Pycnanthus angolensis and Sphenocentrum jollyanum. Fitoterapia 2008, 79:220-222.

23. R: A Language and Environment for Statistical Computing. http://www.Rproject.org, ISBN: 3-900051-07-0.
24. Henry M, Briolant S, Fontaine A, Mosnier J, Baret E, Amalvict R, Fusai T, Fraisse L, Rogier C, Pradines B: In vitro activity of ferroquine is independent of polymorphisms in transport protein genes implicated in quinoline resistance in Plasmodium falciparum. Antimicrob Agents Chemother 2008, 52:2755-2759.

25. Ritchie GY, Mungthin M, Green JE, Bray PG, Hawley SR, Ward SA: In vitro selection of halofantrine resistance in Plasmodium falciparum is not associated with increased expression of Pgh1. Mol Biochem Parasitol 1996, 83:35-46.

26. Sargeant TJ, Marti M, Caler E, Carlton JM, Simpson K, Speed TP, Cowman AF: Lineage-specific expansion of proteins exported to erythrocytes in malaria parasites. Genome Biol 2006, 7:R12.

27. Asahi $\mathrm{H}$, Tolba ME, Tanabe M, Ohmae H: Molecular factors that are associated with early developmental arrest of intraerythrocytic Plasmodium falciparum. Can J Microbiol 2013, 59:485-493.

28. Moura IC, Wunderlich G, Uhrig ML, Couto AS, Peres VJ, Katzin AM, Kimura EA: Limonene arrests parasite development and inhibits isoprenylation of proteins in Plasmodium falciparum. Antimicrob Agents Chemother 2001 45:2553-2558

29. Goodman CD, McFadden Gl: Targeting apicoplasts in malaria parasites. Expert Opin Ther Targets 2013, 17:167-177.

30. Aoki K, Tanaka N, Kusakabe Y, Fukumi C, Haga A, Nakanishi M, Kitade Y, Nakamura KT: Crystallization and preliminary X-ray crystallographic study of phosphoglucose isomerase from Plasmodium falciparum. Acta Crystallogr Sect F: Struct Biol Cryst Commun 2010, 66:333-336.

31. Bozdech Z, Llinas M, Pulliam BL, Wong ED, Zhu J, DeRisi JL: The transcriptome of the intraerythrocytic developmental cycle of Plasmodium falciparum. PLoS Biol 2003, 1:E5.

32. Balu B, Maher SP, Pance A, Chauhan C, Naumov AV, Andrews RM, Ellis PD, Khan SM, Lin JW, Janse CJ, Rayner JC, Adams JH: CCR4-associated factor 1 coordinates the expression of Plasmodium falciparum egress and invasion proteins. Eukaryot Cell 2011, 10:1257-1263.

33. Ross-Macdonald PB, Graeser R, Kappes B, Franklin R, Williamson DH: Isolation and expression of a gene specifying a cdc2-like protein kinase from the human malaria parasite Plasmodium falciparum. Eur J Biochem 1994, 220:693-701.

34. Iwanaga T, Sugi T, Kobayashi K, Takemae H, Gong H, Ishiwa A, Murakoshi F, Recuenco FC, Horimoto T, Akashi H, Kato K: Characterization of Plasmodium falciparum cdc2-related kinase and the effects of a CDK inhibitor on the parasites in erythrocytic schizogony. Parasitol Int 2013, 62:423-430

35. El Bakkouri M, Pow A, Mulichak A, Cheung KL, Artz JD, Amani M, Fell S, de Koning-Ward TF, Goodman CD, McFadden Gl, Ortega J, Hui R, Houry WA: The Clp chaperones and proteases of the human malaria parasite Plasmodium falciparum. J Mol Biol 2010, 404:456-477.

36. Pesce ER, Cockburn IL, Goble JL, Stephens LL, Blatch GL: Malaria heat shock proteins: drug targets that chaperone other drug targets. Infect Disord Drug Targets 2010, 10:147-157.

37. Gitau GW, Mandal P. Blatch GL, Przyborski J, Shonhai A: Characterisation of the Plasmodium falciparum Hsp70-Hsp90 organising protein (PfHop). Cell Stress Chaperones 2012, 17:191-202.

38. Haeggstrom M, VONE A, Kironde F, Fernandez V, Wahlgren M: Characterization of Maurer's clefts in Plasmodium falciparum-infected erythrocytes. Am J Trop Med Hyg 2007, 76:27-32.

39. Winter G, Kawai S, Haeggstrom M, Kaneko O, von Euler A, Kawazu S, Palm D, Fernandez V, Wahlgren M: SURFIN is a polymorphic antigen expressed on Plasmodium falciparum merozoites and infected erythrocytes. J Exp Med 2005, 201:1853-1863.

40. Alexandre JS, Yahata K, Kawai S, Torii M, Kaneko O: PEXEL-independent trafficking of Plasmodium falciparum SURFIN4.2 to the parasite-infected red blood cell and Maurer's clefts. Parasitol Int 2011, 60:313-320.

41. Mphande FA, Ribacke U, Kaneko O, Kironde F, Winter G, Wahlgren M: SURFIN4.1, a schizont-merozoite associated protein in the SURFIN family of Plasmodium falciparum. Malar I 2008, 7:116.

42. Gornicki P: Apicoplast fatty acid biosynthesis as a target for medical intervention in apicomplexan parasites. Int J Parasitol 2003, 33:885-896.

43. Krishna S, Eckstein-Ludwig U, Joet T, Uhlemann AC, Morin C, Webb R, Woodrow C, Kun JF, Kremsner PG: Transport processes in Plasmodium falciparum-infected erythrocytes: potential as new drug targets. Int J Parasitol 2002, 32:1567-1573. 
44. Gimenez-Oya V, Villacanas O, Fernandez-Busquets X, Rubio-Martinez J, Imperial S: Mimicking direct protein-protein and solvent-mediated interactions in the CDP-methylerythritol kinase homodimer: a pharmacophore-directed virtual screening approach. J Mol Model 2009, 15:997-1007.

45. Li G, Basagoudanavar SH, Gowda DC: Effect of GPI anchor moiety on the immunogenicity of DNA plasmids encoding the 19-kDa C-terminal portion of Plasmodium falciparum MSP-1. Parasite Immunol 2008, 30:315-322.

46. Awah NW, Troye-Blomberg M, Berzins K, Gysin J: Mechanisms of malarial anaemia: potential involvement of the Plasmodium falciparum low molecular weight rhoptry-associated proteins. Acta Trop 2009, 112:295-302.

47. Sterkers Y, Scheidig C, da Rocha M, Lepolard C, Gysin J, Scherf A: Members of the low-molecular-mass rhoptry protein complex of Plasmodium falciparum bind to the surface of normal erythrocytes. J Infect Dis 2007, 196:617-621.

48. Tsarukyanova I, Drazba JA, Fujioka H, Yadav SP, Sam-Yellowe TY: Proteins of the Plasmodium falciparum two transmembrane Maurer's cleft protein family, PfMC-2TM, and the $130 \mathrm{kDa}$ Maurer's cleft protein define different domains of the infected erythrocyte intramembranous network. Parasitol Res 2009, 104:875-891.

49. Lavazec C, Sanyal S, Templeton TJ: Hypervariability within the Rifin, Stevor and Pfmc-2TM superfamilies in Plasmodium falciparum. Nucleic Acids Res 2006, 34:6696-6707.

50. Lavazec C, Sanyal S, Templeton TJ: Expression switching in the stevor and Pfmc-2TM superfamilies in Plasmodium falciparum. Mol Microbiol 2007, 64:1621-1634

51. Ward P, Equinet L, Packer J, Doerig C: Protein kinases of the human malaria parasite Plasmodium falciparum: the kinome of a divergent eukaryote. BMC Genomics 2004, 5:79.

52. Schneider AG, Mercereau-Puijalon O: A new Apicomplexa-specific protein kinase family: multiple members in Plasmodium falciparum, all with an export signature. BMC Genomics 2005, 6:30.

53. Oyelade J, Ewejobi I, Brors B, Eils R, Adebiyi E: Computational identification of signalling pathways in Plasmodium falciparum. Infect Genet Evol 2011, 11:755-764.

54. Nunes MC, Okada M, Scheidig-Benatar C, Cooke BM, Scherf A: Plasmodium falciparum FIKK kinase members target distinct components of the erythrocyte membrane. PLoS One 2010, 5:e11747.

55. Sachanonta N, Chotivanich K, Chaisri U, Turner GD, Ferguson DJ, Day NP, Pongponratn E: Ultrastructural and real-time microscopic changes in $P$. falciparum-infected red blood cells following treatment with antimalarial drugs. Ultrastruct Pathol 2011, 35:214-225.

56. Laurent V, Buffet S, Jauréguiberry S, Bruneel F: Pathophysiology of Plasmodium falciparum malaria: main pathways and recent progress. La lettre de I'Infectiologue 2012, XXVII:222-226.

57. Briolant S, Almeras L, Belghazi M, Boucomont-Chapeaublanc E, Wurtz N, Fontaine A, Granjeaud S, Fusai T, Rogier C, Pradines B: Plasmodium falciparum proteome changes in response to doxycycline treatment. Malar J 2010, 9:141.

58. Painter HJ, Morrisey JM, Vaidya AB: Mitochondrial electron transport inhibition and viability of intraerythrocytic Plasmodium falciparum. Antimicrob Agents Chemother 2010, 54:5281-5287.

59. Goodman CD, Su V, McFadden Gl: The effects of anti-bacterials on the malaria parasite Plasmodium falciparum. Mol Biochem Parasitol 2007, 152:181-191.

60. Gysin J, Fandeur T: Saimiri sciureus (karyotype 14-7): an alternative experimental model of Plasmodium falciparum infection. Am J Trop Med Hyg 1983, 32:461-467.

61. Desgrouas C, Desbordes M, Dormoi J, Ollivier E, Parzy D, Taudon N: Quantitative analysis of cepharanthine in plasma based on semiautomatic microextraction by packed sorbent combined with liquid chromatography. J Anal Methods Chem 2014, 2014:695231.

62. Hao G, Liang H, Li Y, Li H, Gao H, Liu G, Liu Z: Simple, sensitive and rapid HPLC-MS/MS method for the determination of cepharanthine in human plasma. J Chromatogr B Analyt Technol Biomed Life Sci 2010, 878:2923-2927.

\section{Submit your next manuscript to BioMed Central and take full advantage of:}

- Convenient online submission

- Thorough peer review

- No space constraints or color figure charges

- Immediate publication on acceptance

- Inclusion in PubMed, CAS, Scopus and Google Scholar

- Research which is freely available for redistribution 